\title{
Probabilistic dynamics of mistuned bladed disc systems using Subset Simulation
}

\author{
Jie Yuan ${ }^{1}$, Giuliano Allegri ${ }^{1,2}$, Fabrizio Scarpa ${ }^{1,3^{*}}$, Ramesh Rajasekaran $^{4}$, Sophoclis Patsias ${ }^{4}$ \\ ${ }^{1}$ Aerospace Engineering, University of Bristol, BS8 1TR Bristol, UK \\ ${ }^{2}$ Department of Aeronautics, Imperial College London, London SW7 2AZ, UK \\ ${ }^{3}$ Dynamics and Control Research Group, University of Bristol, BS8 1TR Bristol, UK \\ ${ }^{4}$ Mechanical Methods, Rolls-Royce plc, PO Box 31, DE24 8BJ Derby, UK
}

\begin{abstract}
The work describes an assessment of subset simulation (SubSim) techniques to increase the computational efficiency for the predictions of probabilistic dynamic behaviour in mistuned bladed disc systems. SubSim is an adaptive stochastic procedure to efficiently compute small failure probabilities, which are expressed as a product of large conditional failures probabilities by introducing intermediate failure events. The original version of SubSim with a classical modified Markov chain Monte Carlo (MCMC) method is used in this work to generate samples related to intermediate failure events. A 2-DOFs model with lumped parameters identified from a high-fidelity finite element model is used to represent a bladed disc. The statistics associated to the maximum forced frequency response amplitudes are evaluated from different levels of the blade mistuning using stiffness perturbations of the blades. Direct Monte Carlo simulations (MCS) are used to benchmark the results from the SubSim. The proposed methodology is shown to capture efficiently the statistical properties of the mistuned blades with less than 5\% samples compared to the direct MCS method. Trade-off parametric studies of the SubSim method indicate that 2000 samples at each level yield an overall good computational efficiency and accuracy for the bladed disk system considered in this work. The study confirms that SubSim techniques can be effectively used in stochastic analysis of bladed disc systems with uncertainty related to the blade configurations.
\end{abstract}

Key words: Subset simulation, mistuned bladed disc system, Uncertainty 


\section{Introduction}

A bladed disk typically consists in a set of disk/blade sectors designed to be identical to each other. This is however an ideal situation, because uncertainties associated to manufacturing, assembly tolerances and wear and tear create uneven distributions of the mechanical properties in individual blades, as well as in the disk. Dimensional and material uncertainties lead to departures of the blade natural frequencies from their nominal design values. This phenomenon is generally denoted as blade mistuning. Mistuning in the free response splits the repeated natural frequencies associated with circumferential modes and distorts the corresponding mode shapes [1-5]. At the same time, the circumferential mode shapes increase the harmonic content of nodal diameters, leading to coupling with engine-induced vibrations [6]. In the worst case scenario, mistuning also causes mode localization phenomena, for which the vibrational energy is transferred and confined to only a few blades in the rotor. This may result in dynamic deformations significantly larger than those estimated at design stage [7,8]. Mistuning therefore compromises the high-cycle fatigue resistance of bladed disks, reducing the endurance and reliability of the whole engine. It is therefore important to predict accurately the effects on the maximum dynamic response of any of the blades in the assembly due to the uncertainties described above [9]. The high-percentile blade forced response obtained from stochastic analysis can be effectively used as an indicator to quantify the influence of such uncertainties [9].

Bladed disks in turbomachinery rotors are commonly represented using detailed finite element models with dimensions well in excess of millions of DOFs. The size of these models can make the dynamic analysis of bladed disks with uncertainties significantly expensive, even when considering the power of modern computational hardware facilities. A cyclical symmetry analysis is usually employed for the efficient evaluation of the free and forced structural responses in tuned systems. However, fullscale finite element models have to be used for mistuned bladed discs due to the asymmetrical nature of the overall response. These models are essentially extremely CPU intensive for the stochastic analysis of mistuned systems in computational grids that are not exoscale. As a consequence, various FE-based reduced order methods (ROMs) have been developed during the last two decades. The current state of the art ROMs used in mistuning applications can be broadly classified into two groups based on the modal reduction techniques used in their development. The first group dating between 1983 and 2000 consists essentially in Component Mode Synthesis (CMS) techniques [10-12]. CMS approaches assume that the blades and the disk are distinct sub-structural elements, thus their modes are separately calculated via deterministic FE analyses. The second group of ROMs was initially introduced by Yang and Griffin in 2001 [13], and they are denoted as System Mode based Methods (SMM). These include the Fundamental Mistuning Model [14], the Component Mode Mistuning [15] and the Integral Mode Mistuning [1]. Lumped parameter models made from cyclic chains of spring- 
mass oscillators are commonly used to represent bladed discs. These simple models have been widely employed during the design stage in preliminary mistuning analysis since they can capture the essence of the existing in the bladed discs and require lower CPU times to run [6]. The lumped parameter models has also shown their suitability to investigate the mistuned forced response characteristics of bladed disks with stochastic distributions of uncertainties within their parameters $[6,9]$

MCS are commonly used in stochastic analysis of mistuned bladed disc systems for their computational robustness[16]. The main drawback of MCS is that it cannot yield small failure probabilities with a limited number of samples. Using MCS, failure probabilities smaller than $1 / \mathrm{N}$ cannot be evaluated with $\mathrm{N}$ samples, and a significantly higher number of samples have to be used to obtain a reliable estimation of a small probability event. For full-scale structures with a large number of degrees of freedom (DOFs), the computational costs increase significantly, sometimes reaching unacceptable levels. As a result, alternative simulations techniques have been used in mistuning, like traditional points-based methods (including both first and second reliability methods[17, 18]) and advanced mean value procedures [19]. However, these techniques fail to predict the distribution of maximum amplitudes from those associated with the stochastic variables, because of the non-convex performance function commonly used to define mistuning problems[9]. Alternatively, response surface methods are capable to approximate the highly nonlinear response surfaces in mistuning problems, but they require using very large numbers of sampling points [20]. Accelerated MCS has been widely used in mistuning analysis, its computational advantage being the fit to an assumed probabilistic distribution with few realizations from MCS [21]. Much debate has focused on the suitable type of distribution to represent the maximum blade forced response, being either a Weibull [22-24] or a Gaussian one [25]. Sanliturk et al have concluded that there is no unique distribution to describe blade amplitudes under different engine orders of excitation, and that accelerated MCS is therefore not a general approach to predict statistics in mistuning problems [26]. Importance Sampling (IS) is another effective technique to reduce the computational cost of stochastic predictions. The principle of this method is to shift the underlying distribution towards the failure region in order to gain information from rare events. The success of IS very depends on a careful choice of the importance sampling density distribution, which undoubtedly requires prior knowledge of the system in the failure region. The method works extremely well when applied to static and dynamic problems with a relatively low-dimensional structural problem. This is because when the dimension of the uncertain parameter space is not too large, the failure region is relatively simple to describe. However, for large structural problems, IS is generally shown to have poor efficiency, as demonstrated by Lee [9] using a radius-based IS method [27] for mistuning analysis. This is due to the large number of stochastic variables that influence the mistuning behaviour and the associated lack of "a priori" knowledge of the system failure region. Chan [28] recently proposed a new procedure to overcome the lack of such pre-knowledge for mistuning problems. He firstly used an optimisation analysis for 
identifying the failure region and then employed IS to characterise the corresponding conditional probabilities. The method is able to estimate very small conditional probabilities, but the identification step of the failure region is still computationally demanding.

Regarding the reliability analysis of engineering systems, Au et.al [29] proposed the SubSim technique for efficiently computing small failure probabilities. The basic idea is to express the failure probability as a product of larger conditional failure probabilities by introducing intermediate failure events. With a proper choice of these intermediate events, the conditional failure probabilities can be made sufficiently large and thus estimated by means of simulations with a small number of samples. The advantage of the SubSim consists in its robustness with respect to dimension size and efficiency on small probabilities estimation. Most importantly, pre-knowledge of the system is not required [29], which makes this method a strong candidate for the stochastic analysis of mistuned bladed disc systems. The application of SubSim techniques in blade/disk assemblies requires the definition of a failure condition. In general, high-cycle fatigue endurance can be expressed using a power law of the vibration amplitudes [30]. Therefore failure can be considered to occur whenever the vibration amplitude associated to a characteristic mode in a mistuned system exceeds significantly the one of its tuned counterpart. In this paper, the focus is placed upon the estimation of the probability of large amplitude vibrations occurring in mistuned system, which will be considered as being the failure events, albeit no detailed fatigue endurance calculation will be carried out. This choice is motivated by the fact that, within the context of this paper, a lumped parameter model will is used to represent the overall rotor dynamics of the disk assembly, whereas endurance calculations would require detail knowledge of the local stress/strain field within the system.

The original version of SubSim makes use of the MCMC [30] technique, based on the modified Metropolis-Hastings algorithm [31, 32], for generating conditional samples. Two newer variants to generate conditional samples have recently been developed for the causality exploration of dynamical systems. SubSim/Splitting is the first variant that is applicable to first-passage problems involving deterministic dynamical systems subjected to stochastic excitation. It makes use of trajectory splitting for generating conditional samples. Another variant, SubSim/Hybrid, uses a combined MCMC/Splitting strategy and so has the advantages of both MCMC and splitting; it is applicable to both uncertain and deterministic dynamical systems. These newer versions of SubSim use the same adaptive procedure as the original technique to generate conditional samples for higher levels based on those from lower levels. The performance of these three SubSim methods have been compared in Ref [17]. All three SubSim variants are proved effectively in problems with large numbers of stochastic values. The two variants using splitting are not considerably superior to the original version.

To the best knowledge of the authors, the use of SubSim techniques in mistuning problem has not been investigated in open literature. This paper describes a feasibility study to assess the use of 
SubSim simulation techniques to improve the computational efficiency of the prediction of probabilistic dynamic behaviour in mistuned bladed disc systems. The bladed disc is represented by a 2-DOFs model with lumped parameters identified from a full-scale finite element model. The procedure to implement the original version of SubSim with classical MCMC techniques is then illustrated. After that, the direct MCS method using new convergent criteria, as the benchmark to the SubSim method, is described and applied in the mistuning problem. The statistics associated to the maximum forced frequency response amplitudes from both SubSim and MCS methods are then evaluated, compared and discussed, followed by a trade-off parametric study to choose the optimum conditional sample size in the SubSim method.

\section{Representative model for a bladed disc system}

Figure 1 show the case study used in this work, which consists in a bladed disc with 24 blades. The lumped parameter model of the disc is made with two DOFs per sector, as done in other uncertainty propagation analyses $[18,19]$. Figure 2 shows the layout of the lumped parameter model used, with one DOF representing the blade motion and the other one representing the disc motion. The blade DOF is associated to the stiffness $k^{b}$ and mass $m^{b}$. The parameters $k^{d}$ and $m^{d}$ represent the stiffness and mass of the disc, respectively. The stiffness $k^{c}$ stands for the coupling between the blades through the disc. The generalised coordinates $q_{i}^{b}, q_{i}^{d}$ are the dynamic responses of the blade and the disc at the $i^{\text {th }}$ sector respectively. Engine order excitation is applied on the blade DOFs. If one assumes harmonic oscillations occurring to the whole system and the presence of structural damping, the equations of motions of the blade-disk system can be written in a matrix form as:

$$
\left[-\omega^{2} \boldsymbol{M}+(1+\eta i) \boldsymbol{K}\right] \boldsymbol{q}=\boldsymbol{F}
$$

In Eq. (1), $\eta$ is the structural damping factor, $\boldsymbol{M}, \boldsymbol{K}$ are the global mass and stiffness matrices, while $\boldsymbol{q}$ and $\boldsymbol{F}$ represents the vectors of the dynamic displacements and external generalized forces respectively. The formulation of these matrices is detailed in $[6,9]$.

This work associates the presence of uncertainty distributions only to the stiffness of the blade.The equation of motion of the system in Eq.(1) is therefore modified by introducing the nominal mass and stiffness matrices $\boldsymbol{M}_{\mathbf{0}}, \boldsymbol{K}_{\mathbf{0}}$ and a global perturbation of the stiffness matrix $\Delta \boldsymbol{K}$, which can be expressed as:

$$
\begin{gathered}
{\left[-\omega^{2} \boldsymbol{M}_{\mathbf{0}}+(1+\eta i)\left(\boldsymbol{K}_{\mathbf{0}}+\Delta \boldsymbol{K}\right)\right] \boldsymbol{q}=\boldsymbol{F}} \\
\Delta \boldsymbol{K}=\left[\begin{array}{cc}
\Delta \boldsymbol{K}^{\boldsymbol{b}} & -\Delta \boldsymbol{K}^{\boldsymbol{b}} \\
-\Delta \boldsymbol{K}^{\boldsymbol{b}} & \Delta \boldsymbol{K}^{\boldsymbol{b}}
\end{array}\right], \Delta \boldsymbol{K}^{\boldsymbol{b}}=\operatorname{diag}\left(\Delta k_{1}^{b}, \Delta k_{2}^{b}, \cdots, \Delta k_{N_{b}}^{b}\right), \Delta k_{i}^{b}=\delta_{i}^{b} k^{b}
\end{gathered}
$$


$\Delta \boldsymbol{K}^{\boldsymbol{b}}$ represents a perturbation matrix depending on the stiffness of the blades only, with $\delta_{\mathrm{i}}^{\mathrm{b}}$ giving the perturbation of the stiffness for the $i^{\text {th }}$ blade from its nominal value $k^{b}$. The values of the perturbation are randomly generated according to a log-normal distribution, which guarantees the presence of positive values of the spring stiffness. It is worth noticing that a normal distribution cannot be used because it may lead to the presence of negative spring stiffness, which may be misleading in a mistuning reliability analysis especially for cases focusing on the tail of the distribution (i.e., small probabilities).

The reference finite element model of the bladed disc shown in Figure 1 is built using the commercial software ANSYS Rel. 11.0 (Ansys Inc.,2008). The model consists in 1344 3D SOLID95 8-node elements. Each node has three translational DOFs. The total number of DOFs is 10,752. The rotor is entirely made of titanium, considered as a homogenous and isotropic material (Young's modulus of $115 \mathrm{GPa}$, density of $4800 \mathrm{~kg} / \mathrm{m} 3$ and Poisson's ratio of 0.32 ). The blade has a high slenderness ratio (10), and a uniform rectangular cross section. The model is clamped at the inner circle of the disc by zeroing all DOFs. A modal analysis has been performed on the finite element model using a Block Lanczos method on using a PC with an Intel $3.2 \mathrm{GHz}$ dual-core processor and 4 GB RAM.

Figure 3 shows the variations of the first six natural frequencies (NFs) against the nodal diameters (NDs) of the finite element model that characterizes the free vibration of a bladed disc. The fact that the disc itself becomes more rigid with the increasing NDs makes the NFs of the rotor gradually increase and finally approach the NFs of the blade alone. The horizontal lines at high NDs represent the blade-dominated modes, while the slope-varying lines at low NDs represent disk-dominated ones. The veering regions in between indicate some significant disk-blade interactions which have an important effect on the forced frequency response of mistuned systems[6]. From Figure 3, it is possible to observe that the $2^{\text {nd }}$ and $3^{\text {rd }}$ out-of-plane flapping modes exhibit significant changes of the NFs within specific intervals of the NDs. This suggests the existence of inter-blade coupling in these modes that facilitates the energy transfer between the blades through the disc. Therefore this mechanism can generate mode localization and extremely high forced frequency responses. Therefore, the $2^{\text {nd }}$ and $3^{\text {rd }}$ out of plane flapping modes are among the most interesting mode shapes for mistuning analysis.

The parameters of the lumped model are identified from the response of the full scale FE model based on the geometric characteristics of the natural frequency curves in the veering region. It is worth noticing that this parametric identification is not aimed at capturing the specific modes from the fullscale finite element model, but tends to identify the variation of the natural frequencies in the veering regions, which are more significant for mistuning analysis purposes. This fitting process follows the methodology proposed in [20] to identify the main lumped stiffness and mass parameters 
$\left(k^{b}, k^{c}, k^{d}, m^{d}, m^{b}\right)$. Setting the concentrated stiffness and mass parameters $k^{b}$ and $m^{b}$ to unity, the lumped parameter model is expressed in terms of non-dimensional stiffness and mass variables, as shown in Table 1. A non-dimensional frequency $\xi=\omega / \omega_{0}$ is also introduced, for which $\omega_{0}$ corresponds to the natural frequency of mode of the blade alone in the lumped parameter model calculated with the values given in Table 1 and $2^{\text {nd }}$ flap mode in the finite element model at $12^{\text {th }}$ nodal diameter respectively. Making use of this non-dimensional frequency, Figure 4 shows the direct comparison of the variation of the NFs with respect to the NDs between the lumped parameter and the FE model. The two models show a general good agreement, in particular in the veering region at low NDs (as expected). It can be therefore concluded that the lumped parameter model is an adequate representation of the dynamic behaviour of the two dominating flapping modes in the bladed disc system.

Early high cycle fatigue failure of blades is mainly attributed to extremely high dynamic responses in the bladed disc system [28]. Therefore, a physical quantity of great interest here is the maximum amplitude of the dynamic response for any of the blades across a specific frequency range. The maximum amplitude factor used in this case study is defined as the ratio between the largest responses of the forced vibration in a given mistuned bladed disc to the one found in a tuned counterpart under the same type of excitation. In the present analysis, the $2^{\text {nd }}$ order engine excitation is selected so that the veering region can be excited. The non-dimensional frequency bandwidth used is normalized by the first natural frequency of the tuned rotor at the $2^{\text {nd }}$ nodal diameter to include the resonance frequency point of the blade mode. The structural damping factor is set at $0.5 \%$ [20]. Figure 5 shows the frequency response of 24 blades in a mistuned system from the lumped parameter model, where the maximum amplification factor is about 1.4. Previous works have demonstrated that the distribution of the maximum amplitude factor is significantly dependent upon the distribution of the mistuning patterns on the blades [33]. The high percentile of the amplitude factor can be reliably predicted when one has a good knowledge of the distribution of the mistuned blades. However, in practice the statistical distribution associated to the properties of the blades is not well known, mainly due to the significant costs involved in the determination of these properties using full-scale bladed disc in a Design of Experiments (DoE) environment. Because of the level of uncertainties associated to the distribution of the blade properties, the focus of the estimated high percentile of the maximum amplitude factor is within the range between $95^{\text {th }}$ and $99.5^{\text {th }}[6,9,33,34]$, i.e. at failure probability levels up to $10^{-3}$.

\section{Subset simulation with MCMC}


As previously discussed, SubSim is an adaptive stochastic procedure that allows to compute efficiently small failure probabilities. This technique is based on the fact that a small failure probability can be expressed as a product of large values of conditional failure probabilities by introducing several intermediate failure events. The vibration-induced failure of the blade under high cycle fatigue is here considered as the key reliability indicator for mistuning problem [33]. Therefore, the probability of exceeding some given vibration amplitude levels is considered as an indication of "failure" in the analysis that follows.

Let $\mathrm{F}$ denote the failure domain and $\mathrm{F}_{\mathrm{i}}, \mathrm{i}=1, \ldots, \mathrm{m}$ are the intermediate events which can be expressed as:

$$
F_{i}=\left\{H>h_{i}\right\}, i=1, \ldots, m, h_{1}<h_{2}<\cdots<h_{m}
$$

In Eq.(4), $\mathrm{H}$ is the response function of the maximum amplitude factor; $h_{1}, h_{2}, \ldots, h_{m}$ is a sequence of amplitude thresholds each representing an intermediate event. Accordingly, the intermediate failure region $F_{i}$ can be arranged such that $F_{1} \supset F_{2} \supset \cdots \supset F_{m}=F$ in order to form a decreasing sequence of failure events. The failure probability $\mathrm{P}_{\mathrm{f}}$ can be evaluated as the conditional probability of the last subset $F_{m}$ given that for $F_{m-1}$ and the probability of the event belonging to the subset $F_{m-1}$. This process can be recursively repeated as:

$$
P_{f}=P\left(F_{m} \mid F_{m-1}\right) P\left(F_{m-1}\right)=\cdots=P\left(F_{1}\right) \prod_{i=2}^{m} P\left(F_{i} \mid F_{i-1}\right)
$$

Eq. (5) shows that $\mathrm{P}_{\mathrm{f}}$ can be efficiently evaluated as the product of several conditional probabilities, in lieu of calculating $\mathrm{P}_{\mathrm{f}}$ directly. However, the optimum intermediate threshold values are difficult to determine for different conditional levels. Intermediate thresholds are therefore chosen "adaptively" so that each of the conditional probabilities is approximately equal to a common specified value.

To evaluate $\mathrm{P}_{\mathrm{f}}$ in Eq. (11), $\mathrm{P}\left(\mathrm{F}_{1}\right)$ and $\mathrm{P}\left(\mathrm{F}_{\mathrm{i}} \mid \mathrm{F}_{\mathrm{i}-1}\right)$ need to be computed. The unconditional probability $\mathrm{P}\left(\mathrm{F}_{1}\right)$ for the first subset can be readily estimated using MCS. However, in order to reduce the computational effort involved in using MCS, a Markov Chain MCS technique based on the modified Metropolis-Hastings (M-H) algorithm is used herein to generate efficiently samples that satisfy the prescribed conditional probability. The modified $\mathrm{M}-\mathrm{H}$ algorithm can overcome the difficulties of its original version to sample the rare evens in the high dimensional problems. More details regarding the modified M-H algorithm can be found in references [29, 31, 32]. It is worth noting that the probability distribution selected in the algorithm governs the choice of the candidate samples and, consequently, the overall computational cost of the M-H algorithm [29].

The parameters for the SubSim method have to be set before the actual simulation of the mistuning problem. These consist of the conditional probability $P_{\mathrm{o}}$, the number of levels $\mathrm{m}$, and the number of samples for each level $N_{T}$, as well as the probability density function (PDF) for the M-H algorithm. 
$P_{o}=0.1$ is chosen for the case study considered here and, as discussed by Au and Beck [29], this value of conditional probability is regarded as a reasonable choice. A constant number of samples $N(1500$ in our case) is considered for each different conditional levels. The number of levels $\mathrm{m}$ is expressed as:

$$
\mathrm{m}=\frac{\log \left(\mathrm{P}_{\mathrm{f}}\right)}{\log \left(\mathrm{P}_{\mathrm{o}}\right)}
$$

The value of $\mathrm{m}$ is dependent on the chosen reliability level. In principle the level sample sizes $N_{T}$ cannot be determined "a priori". However, a coarse initial estimation originally proposed in Ref [29] can be used:

$$
N_{T} \approx m N=\left|\log \left(\mathrm{P}_{\mathrm{f}}\right)\right|^{r} \times \frac{(1+r)\left(1-p_{0}\right)}{\mathrm{P}_{\mathrm{o}}\left|\log \left(\mathrm{P}_{\mathrm{o}}\right)\right|^{r} \delta^{2}}
$$

In Eq. (7), the exponent $r$ specifies the correlation of the $P_{\mathrm{i}}$ for each individual subset, while $\delta$ is the variance coefficient associated with the failure probability $P_{f}$. A $\log$ normal distribution with a much smaller standard deviation is used as the assumed PDF in the M-H algorithm.

The procedure for adaptively generating samples of conditional levels is illustrated in Figure 6. For the mistuning problem, the method is implemented according to the following steps:

Step 1: Randomly generate N samples of blade stiffness perturbation vectors by direct MCS for the first unconditional case; evaluate the corresponding maximum amplitude factors for each mistuning pattern; choose an appropriate conditional probability $\mathrm{p}_{0}$.

Step 2: Choose the failure threshold $h_{1}$ such that $\left[\left(1-p_{0}\right) N\right]$ responses fall outside the subset $F_{1}$ and $p_{0} \mathrm{~N}$ samples that belong to $F_{1}$; the samples inside $F_{1}$ are the original conditional samples at level 1 .

Step 3: Implement modified $\mathrm{M}-\mathrm{H}$ algorithm with these original samples to simulate an additional $\left[\left(1-\mathrm{p}_{0}\right) \mathrm{N}\right]$ conditional samples at the conditional level 1 .

Step 4: Similarly, set $h_{2}$ so that $\left[\left(1-p_{0}\right) N\right]$ responses lie out of the subset $F_{2}$; the rest of the $p_{0} N$ samples provide seeds for applying the M-H algorithm to generate the samples for conditional level 2.

Step 5: repeat the procedure above for higher conditional levels until the samples for the specified conditional level $\mathrm{m}$ have been generated; then the failure probability can be evaluated.

\section{Benchmark Monte Carlo Simulations}


The MCS is used to benchmark the results obtained from the SubSim method. The true failure probability $p$ is approximately evaluated by direct MCS via calculating the event function $H\left(x_{i}\right)$ for a number of samples $N$ :

$$
p \approx p_{D M C}=\frac{1}{N} \sum_{i=1}^{N} H\left(x_{i}\right)
$$

In most cases, extremely large values of $N$ have to be selected to ensure convergence, however this may lead to severe computational penalties.

To quantify the convergence properties of direct MCS methods, in this paper it is used the concept of confidence interval. A $100(1-\alpha) \%$ confidence interval means that there is $100(1-\alpha) \%$ chance that the "true" probability will fall within a range $2 \Delta \mathrm{p}$ wide from the estimated value, i.e.:

$$
\mathrm{P}\left(p_{D M C}-\Delta p \leq p \leq p_{D M C}+\Delta p\right)=1-\alpha
$$

In Eq. (9), the half-width of the confidence interval $\Delta \mathrm{p}$ can be evaluated using the sample variance $\sigma_{D M C}^{2}$ and sample size $N$ :

$$
\Delta p=z_{N-1,1-\alpha / 2} \sqrt{\frac{\sigma_{D M C}^{2}}{N}}
$$

In (10), the term $\mathrm{z}$ is the number of DOFs in the student's t-distribution that is dependent on the sample size and the required confidence level. Typically, a 95\% confidence level is taken for reliability analysis, so that $\mathrm{z}$ approaches 1.96 when $\mathrm{N}$ approaches infinity [27]. On the other hand, one can estimate the total number of samples $N$ from Eq. (10) for any given failure probability and confidence level.

By virtue of the law of large numbers, the sample variance $\sigma_{D M C}^{2}$ can be approximated using the following:

$$
\sigma_{D M C}^{2} \approx p_{D M C}\left(1-p_{D M C}\right)
$$

Figure 7 shows the variations of the relative half width $\left(\frac{\Delta p}{p}\right) \%$ with the sample size for different failure probability levels. The relative half width for $p=0.05$ and $p=0.01$ levels is smaller than $25 \%$ when using 10,000 samples. Considering the same number of samples, $\left(\frac{\Delta p}{p}\right) \%$ significantly widens, 
reaching $40 \%$ and $60 \%$ respectively for $p=0.005$ and $p=0.001$ levels. If one assumes that the convergence criterion of the MCS method is satisfied when the relative half width of the $95 \%$ confident interval is less than $20 \%$, the number of samples for $\mathrm{p}=10^{-2}, 10^{-3}$, can be estimated at $10^{4}, 10^{5}$ respectively. In other words, when using the same convergence criterion for each of the MSC runs, the sample size must be one order of magnitude larger than the associated inverse probability.

Figure 8 shows the probability density function (PDF) of the maximum amplitude factors calculated with the representative lumped parameter model using the MCS and considering a $0.5 \%$ coefficient of variance of the blades stiffness for 10,000 samples. By using a log normal distribution to generate blade stiffness perturbations, the perturbation is always larger than-100\%. The maximum amplitude factors vary from 1 to 2.5 , with a mean of about 1.8017 and standard deviation of 0.1592 . The distribution of the maximum amplitude factors has been then fitted using Lognormal, Weibull and a generalized extreme value distribution. The log likelihoods of these three fitting distributions are 4331.4, 3285.02 and 4380.84, respectively. As the higher value of log likelihood indicates better fit, these low values of likelihood indicate the maximum amplitude factors do not accurately follows either the Normal, Webull distributions or Generalized extreme value distribution, a feature that has been already observed by Sanliturk [26]. It can be however noticed that the Generalized extreme value distribution provides the best fitting of the maximum amplitude factors amongst the three distributions used.

The failure distribution of the responses is shown in Figure 9, together with the upper and lower bounds of the $95 \%$ confidence interval. As expected, the half width range gradually expands with decreasing failure probability. The $95 \%$ confidence interval for $10^{-2}$ failure probability of mistuning is between 0.0008 and 0.0012 (the relative half width is $20 \%$, i.e. the MCS convergence criterion is satisfied). However, to estimate small failure probability below $10^{-3}$, the relative half width percentage of 'true' probability widens, reaching about $100 \%$. If the current sample size were used, one would obtain highly unreliable failure probability estimations. Therefore, a sample size at least one order of magnitude larger would be needed to estimate the one order smaller failure probability level (see Figure 7).

\section{Main results and discussions}

Figure 10 shows the failure probability distributions as estimated from the SubSim method and the MCS for the mistuned bladed disc systems. As stated above, a failure probability up to $10^{-3}$ is 
considered because of the uncertainties associated to the distribution of the blade properties in practical cases. Three conditional levels and 1500 samples in each level are used to compute the failure probability using the SubSim technique. The result from the MCS with 100,000 samples provides the benchmark for the SubSim method. The relative half width of the estimated failure probability on the level of $10^{-3}$ is close to $20 \%$.

The green curve in the figure represents the average value of 10 independent SubSim simulations. The SubSim and MSC results (in blue) agree quite well within the range of probability considered, except for the case of extremely low failure probabilities (below $10^{-4}$ ). This is mainly due to the large uncertainty associated to the MCS results, which are not converging for probability values below $10^{-4}$ with the current sample size and the insufficient conditional levels used in the SubSim method. It is worth remarking that the SubSim technique allows estimating the probability down to a value of $10^{-4}$, which indicates that the estimation of the sample size for each conditional level from Eq. (7) is conservative. Nonetheless, the number of samples used in the SubSim method is less than $5 \%$ the one required by using the MCS. This demonstrates the very significant improvement of the computational efficiency provided by the SubSim approach. The results of the SubSim method are also compared with the ones from the MCS with the same sample size (in red). Compared to SubSim, the range of the converged failure probabilities obtained by the MCS is limited to less than $10^{-2}$. In the probability range from $10^{-2}$ to $10^{-3}$ the MCS with a reduced sample size of 4500 shows a significant difference with the benchmark case (100,000 samples).

Figure 11 shows the different percentile amplitude factor of the PDF between the MCS $(100,000$ samples) and the SubSim (3 levels, 1500 samples each) for different perturbation levels of the blade stiffness. In the SubSim method, the ratio between the standard deviation of the PDF used in modified $\mathrm{M}-\mathrm{H}$ algorithm and the variance of the mistuning levels is kept constant at $20 \%$ through the whole sensitivity study. The percentile amplitude factors increase rapidly for a low perturbation level, i.e. below $0.5 \%$. With a further increase of the mistuning level the amplitude factors slightly decrease and then remain virtually constant. The variations of the amplitude factors is qualitatively similar to the one reported in [21], which further confirms the applicability of the current lumped parameter model for statistical investigations of mistuning patterns. Figure 11 also shows that the predictions of the amplitude factor from the SubSim method are always larger than the one predicted by the MCS on the 99 percentile and 99.5 percentile amplitude factors. This fact can be mainly attributed to the large standard deviation of the PDF used for the modified $\mathrm{M}-\mathrm{H}$ algorithm to generate samples in the conditional levels. There is an excellent agreement between the three types of percentiles response from the MCS and the SubSim method, especially on the $95^{\text {th }}$ percentile amplitude factor. With the mistuning level varying from $0.01 \%$ to $2 \%$, the average differences of the amplitude factors between SubSim and MCS for the $99.5^{\text {th }}, 99^{\text {th }}$ and $95^{\text {th }}$ percentiles are $0.8 \%, 0.4 \%$ and $0.2 \%$ respectively. 
Figure 12 also shows the comparison between the relative errors for these four sets of amplitude factors against the MCS results at different mistuning levels. The largest relative errors for the $99.5^{\text {th }}$ and $99^{\text {th }}$ percentiles occur within the most sensitive range of the variance of the amplitude factor perturbation levels (between 0.0006 and 0.004).

When the variance varies from $0.06 \%$ to $0.5 \%$ the amplitude factor of the mistuned system is sharply increased with the glowing mistuning level. The ratio of standard deviation of proposed PDF in modified M-H algorithm to variance of the mistuning level however remains the same during the whole range. This leads to relatively large standard deviation in this sensitive range, and therefore slightly large amplitude factors in difference percentiles - from here the explanation about the larger amplitude factors predicted by SubSim compared to the ones calculated by MCS. Smaller errors would be obtained by decreasing the standard deviation of the proposed PDF in modified M-H algorithm. The standard deviation used in $\mathrm{M}-\mathrm{H}$ algorithm should be adjusted in an adaptive manner with respect to the sensitivity of the amplitude factors versus the mistuning levels

The dependence of the coefficient of variation (c.o.v.) versus the failure probability from 10 sets of independent SubSim runs (4 levels, 6000 samples) is shown in Figure 13. For the comparison, the c.o.v of the MCS estimate are illustrated at a particular failure probability level using the same total number of sample as in SubSim $\left(\mathrm{N}=1500,3000,4500,6000\right.$ for $P_{f}=10^{-1}, 10^{-2}, 10^{-3}, 10^{-4}$, respectively). Therefore, the variability between the two methods can be compared at approximately the same computational effort. The c.o.v. for SubSim almost decreases linearly with the increasing failure probability, while the c.o.v. for MCS decreases almost exponentially. The c.o.v. calculated by the SubSim and MCS methods are almost the same within the 'large' failure probability region (from $10^{-1}$ to $10^{-2}$ ), mainly because the SubSim procedure for relatively large failure probability is computed from an initial MCS run. However, when the failure probability is smaller than $10^{-2}$ one can observe a marked difference between the SubSim results and the MCS simulations. The latter do not converge in probability, while the former do. This is a further indication of the huge computational advantage of the SubSim technique over the MCS when the assumed failure probability becomes smaller. SubSim method would obviously stand out and have a better convergence than MCS if the interested failure probability is less than 0.01 .

\section{Trade-off parametric study}

The number of samples in each conditional level is the key parameter governing the computational cost of the SubSim method. If the sample size is too large, then the computational cost will tend to 
approach the MCS one, whilst an insufficient sample size would lead to poor accuracy in the probability estimation. The coarse estimate of the sample size yielded by Eq. (7) may indeed not be accurate, since it is dependent on the choice of the exponentr. A trade-off study is therefore carried out to investigate the optimum number of samples for the mistuning problem in the case study of this paper. Figure 14 shows the comparisons between c.o.v. related to different sample sizes. The efficiency of the SubSim is strongly improved when the sample size increases from 500 to 2000 in the interested failure probability range from 0.01 to 0.001 . However, for a number of samples above 2000, where the values of the variance cluster between 0.5 and 0.6 the increase of the sample size yields little benefit. Figure 15 shows the comparison of normalized computing time for different conditional sample sizes. The computational expense increases linearly with the sample size, and a sample population of 2000 is therefore chosen as a sensible choice for the case study considered in this paper. This however implies that Eq.(7) can underestimate slightly (20\%) the sample size required for the high number of stochastic variables considered in this work. This problem may be further exacerbated if the number of DOFs is increased.

\section{Conclusions}

The main objective of this work is to investigate the feasibility of applying the SubSim method to mistuned bladed disc systems in order to reduce the computational costs associated to the analysis. The traditional MCS does provide a simple and robust approach for the stochastic analysis of mistuning problems, but the associated computational cost penalties are significant, especially when the estimation of relatively small failure probabilities is sought. The applicability of importance sampling techniques to MCS is limited by the required "a priori" knowledge of the system failure region, which is difficult to characterize in large-scale systems. SubSim is an adaptive stochastic procedure for computing efficiently small failure probabilities without the need of any "a priori" knowledge of the system, and provides the potential to reduce computational costs in mistuning problems. A 2-DOFs model with lumped parameters identified from a finite element model is developed to represent a bladed disc. The original version of SubSim with a classical modified MCMC method is used in this work to simulate samples related to intermediate failure events. The statistics associated to the maximum forced frequency response amplitudes are evaluated from different levels of the blade mistuning introducing stiffness perturbations in the blades.

Direct MCS approaches are used to benchmark the results from the SubSim based analyses. The concept of confidence interval is introduced to quantify the uncertainty of MCS and determine an adequate sample size for convergence in probability. SubSim is shown to capture in an efficient way the statistical properties of the mistuned blades for a wide range of stiffness perturbation levels. The number of samples required by the SubSim approach is less than $5 \%$ of the one required by the MCS method. Considering the same sample size, the two methods have comparable computational costs for 
failure probabilities ranging from $10^{-1}$ to $10^{-2}$. However, when estimating failure probability between $10^{-2}$ to $10^{-3}$, the SubSim method is at maximum twice as efficient as the benchmark MCS. It is also found that the largest relative errors of amplitude factors using SubSim method occur in the most sensitive range of mistuning levels. It suggests that the standard deviation of the probability density function in the modified $\mathrm{M}-\mathrm{H}$ algorithm needs to be adaptively selected with respect to the sensitivity of amplitude factors to mistuning levels. Trade-off parametric studies further indicate that an overall conditional probability level of 0.1 combined with 2000 samples at each intermediate failure level yield an overall accurate solution of the mistuning problem considered here with greatly reduced computational costs. The study confirms that SubSim techniques can be effectively used in stochastic analysis of bladed disc systems with uncertainty dependent on the blades configuration. 


\section{Acknowledgements}

The authors would like to acknowledge the support of Rolls-Royce plc for the support of this research through the Composites University Technology Centre (UTC) at the University of Bristol, UK. Special acknowledgement goes also to the Strategic Investment in Low carbon Engine Technology (SILOET) programme supported by Rolls-Royce plc and Technology Strategy Board (TSB), and to the China Scholarship Council. The authors would also like to thank the Reviewers for their useful suggestions.

\section{Reference}

[1] P. Vargiu, C.M. Firrone, S. Zucca, M.M. Gola, A reduced order model based on sector mistuning for the dynamic analysis of mistuned bladed disks, International Journal of Mechanical Sciences, 53 (2011) 639-646.

[2] D.J. Ewins, The effects of detuning upon the forced vibrations of bladed disks, Journal of Sound and Vibration, 9 (1969) 65-79.

[3] C.H. Hodges, Confinement of vibration by structural irregularity, Journal of Sound and Vibration, 82 (1982) 411-424.

[4] C. Hodges, J. Woodhouse, Vibration isolation from irregularity in a nearly periodic structure: Theory and measurements, The Journal of the Acoustical Society of America, 74 (1983) 894.

[5] C. Pierre, Mode localization and eigenvalue loci veering phenomena in disordered structures, Journal of Sound and Vibration, 126 (1988) 485-502.

[6] M.P. Castanier, C. Pierre, Modeling and Analysis of Mistuned Bladed Disk Vibration: Current Status and Emerging Directions, Journal of Propulsion and Power, 22 (2006) 384-396.

[7] J. MacBain, P. Whaley, Maximum resonant response of mistuned bladed disks, Journal of Vibration Acoustics Stress and Reliability in Design, 106 (1984) 218.

[8] D. Whitehead, The maximum factor by which forced vibration of blades can increase due to mistuning, Journal of Engineering for Gas turbines and Power, 120 (1998) 115-119.

[9] S.-Y. Lee, M.P. Castanier, C. Pierre, Assessment of probabilistic methods for mistuned bladed disk vibration, in: Proceedings of the 46th AIAA/ASME/ASCE/AHS/ASC Structures, Structural dynamics, and Materials Conference, AIAA-2005-1990, 2005.

[10] H. Irretier, Spectral Analysis of Mistuned Bladed Disk Assemblies by Component Mode Synthesis, in: D.J.E.A.V. Srinivasan (Ed.) Vibrations of Bladed Disk Assemblies, American Society of Mechanical Engineers, New York, 1983.

[11] G. Óttarsson, Dynamic modeling and vibration analysis of mistuned bladed disks, in, University of Michigan, 1994.

[12] M.-T. Yang, J. Griffin, A reduced order approach for the vibration of mistuned bladed disk assemblies, Journal of Engineering for Gas turbines and Power, 119 (1997) 161-167.

[13] M.-T. Yang, J. Griffin, A reduced-order model of mistuning using a subset of nominal system modes, Journal of Engineering for Gas turbines and Power, 123 (2001) 893-900.

[14] D.M. Feiner, J. Griffin, A fundamental model of mistuning for a single family of modes, Journal of turbomachinery, 124 (2002) 597-605.

[15] S.-H. Lim, R. Bladh, M.P. Castanier, C. Pierre, Compact, Generalized Component Mode Mistuning Representation for Modeling Bladed Disk Vibration, AIAA Journal, 45 (2007) 2285-2298.

[16] R.Y. Rubinstein, D.P. Kroese, Simulation and the Monte Carlo method, John Wiley \& Sons, 2011. 
[17] S. Au, J. Ching, J. Beck, Application of subset simulation methods to reliability benchmark problems, Structural Safety, 29 (2007) 183-193.

[18] O. Bendiksen, Flutter of mistuned turbomachinery rotors, Journal of Engineering for Gas Turbines and Power, 106 (1984) 25-33.

[19] G. Ottarsson, C. Pierre, A transfer matrix approach to free vibration localization in mistuned blade assemblies, Journal of Sound and Vibration, 197 (1996) 589-618.

[20] S. Baik, M.P. Castanier, C. Pierre, Mistuning sensitivity prediction of bladed disks using eigenvalue curve veerings, in: Proceedings of the 9th National Turbine Engine High Cycle Fatigue Conference, 2004.

[21] M.P.C.C. Pierre, Modeling and Analysis of Mistuned Bladed Disk Vibration:Status and Emerging Directions, JOURNAL OF PROPULSION AND POWER, 22 (2006).

[22] J.L. Neuringer, W. Mcllroy, B. Kshirsagar, J. Nagaraju, M. Krishna Murthy, G. SOGLIERO, A. SRINIVASAN, Fatigue life estimates of mistuned blades via a stochastic approach, AIAA Journal, 18 (1980) 318-323.

[23] M.P. Mignolet, W. Hu, I. Jadic, On the forced response of harmonically and partially mistuned bladed disks. Part II: partial mistuning and applications, International Journal of Rotating Machinery, 6 (2000) 43-56.

[24] M.P. Mignolet, W. Hu, I. Jadic, On the forced response of harmonically and partially mistuned bladed disks. Part I: Harmonic mistuning, International Journal of Rotating Machinery, 6 (2000) 29-41. [25] A. Sinha, Statistics of the peak maximum amplitude of the forced response of a mistuned bladed disk, in, ASME, 2005.

[26] K. Sanliturk, M. Imregun, D. Ewins, Statistical analysis of random mistuning of bladed assemblies, in: INSTITUTION OF MECHANICAL ENGINEERS CONFERENCE PUBLICATIONS, MEDICAL ENGINEERING PUBLICATIONS LTD, 1992, pp. 51-51.

[27] A.M. Law, W.D. Kelton, W.D. Kelton, Simulation modeling and analysis, McGraw-Hill New York, 1991.

[28] Y.-J. Chan, D. Ewins, The amplification of vibration response levels of mistuned bladed disks: its consequences and its distribution in specific situations, Journal of Engineering for Gas Turbines and Power, 133 (2011) 102502.

[29] S.-K. Au, J.L. Beck, Estimation of small failure probabilities in high dimensions by subset simulation, Probabilistic Engineering Mechanics, 16 (2001) 263-277.

[30] P. Lukáš, M. Klesnil, J. Polak, High cycle fatigue life of metals, Materials Science and Engineering, 15 (1974) 239-245.

[31] N. Metropolis, A.W. Rosenbluth, M.N. Rosenbluth, A.H. Teller, E. Teller, Equation of state calculations by fast computing machines, The journal of chemical physics, 21 (2004) 1087-1092. [32] W.K. Hastings, Monte Carlo sampling methods using Markov chains and their applications, Biometrika, 57 (1970) 97-109.

[33] Y.J. Chan, Variability of blade vibration in mistuned bladed discs, in, Imperial College London (University of London), 2009.

[34] J. Li, M.P. Castanier, M. Pierre, S.L. Ceccio, Experimental Monte Carlo mistuning assessment of bladed disk vibration using forcing variations, in: Proceedings of the 47th

AIAA/ASME/ASCE/AHS/ASC Structures, Structural dynamics, and Materials Conference, 2006. 


\section{Figures and Tables}

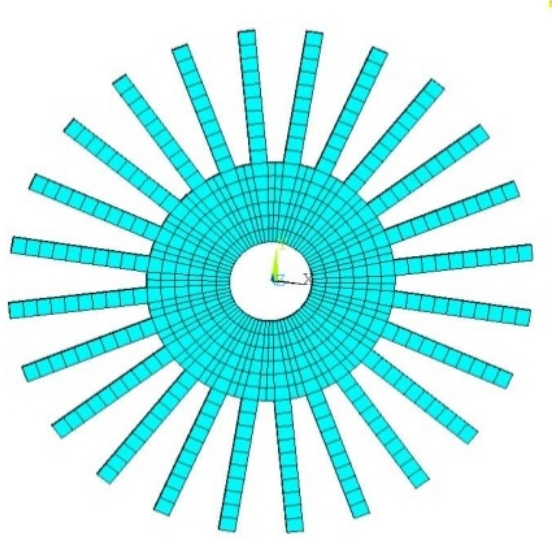

Figure 1 finite element model of a bladed disc

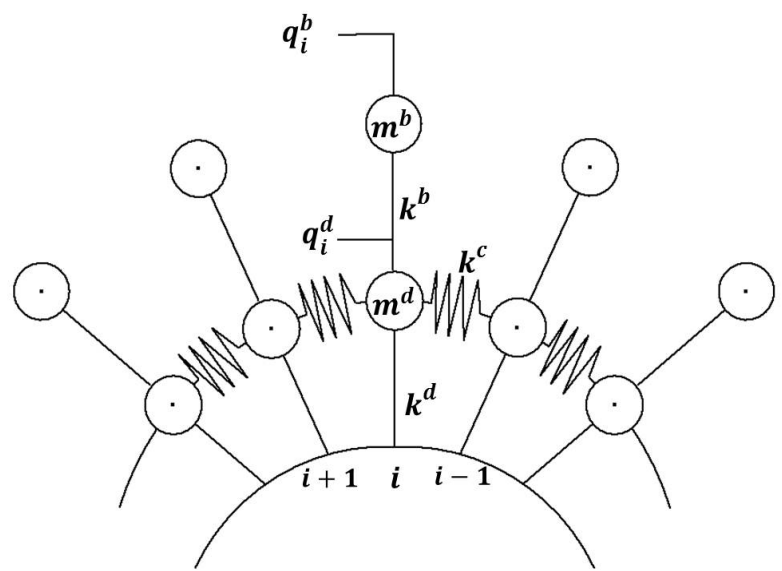

Figure 2. Two DOFs per sector model

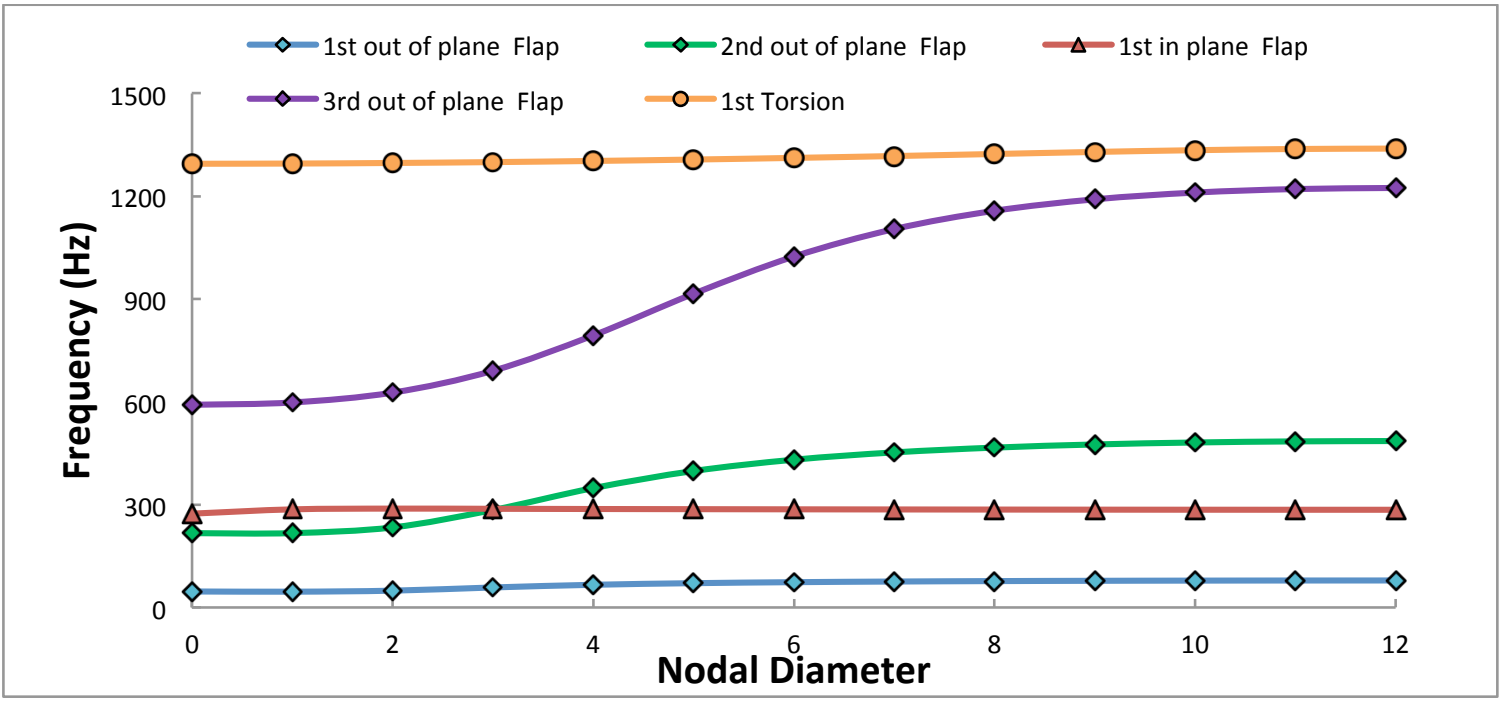

Figure 3 Natural frequencies versus nodal diameters (FE)

Table 1 Parameters identified for lumped parameter model

\begin{tabular}{|c|c|}
\hline Parameters & Non-dimensional Values \\
\hline$k^{d} / k^{b}$ & 32 \\
\hline$k^{c} / k^{b}$ & 1500 \\
\hline
\end{tabular}




\begin{tabular}{|c|c|}
\hline$m^{d} / m^{b}$ & 200 \\
\hline
\end{tabular}

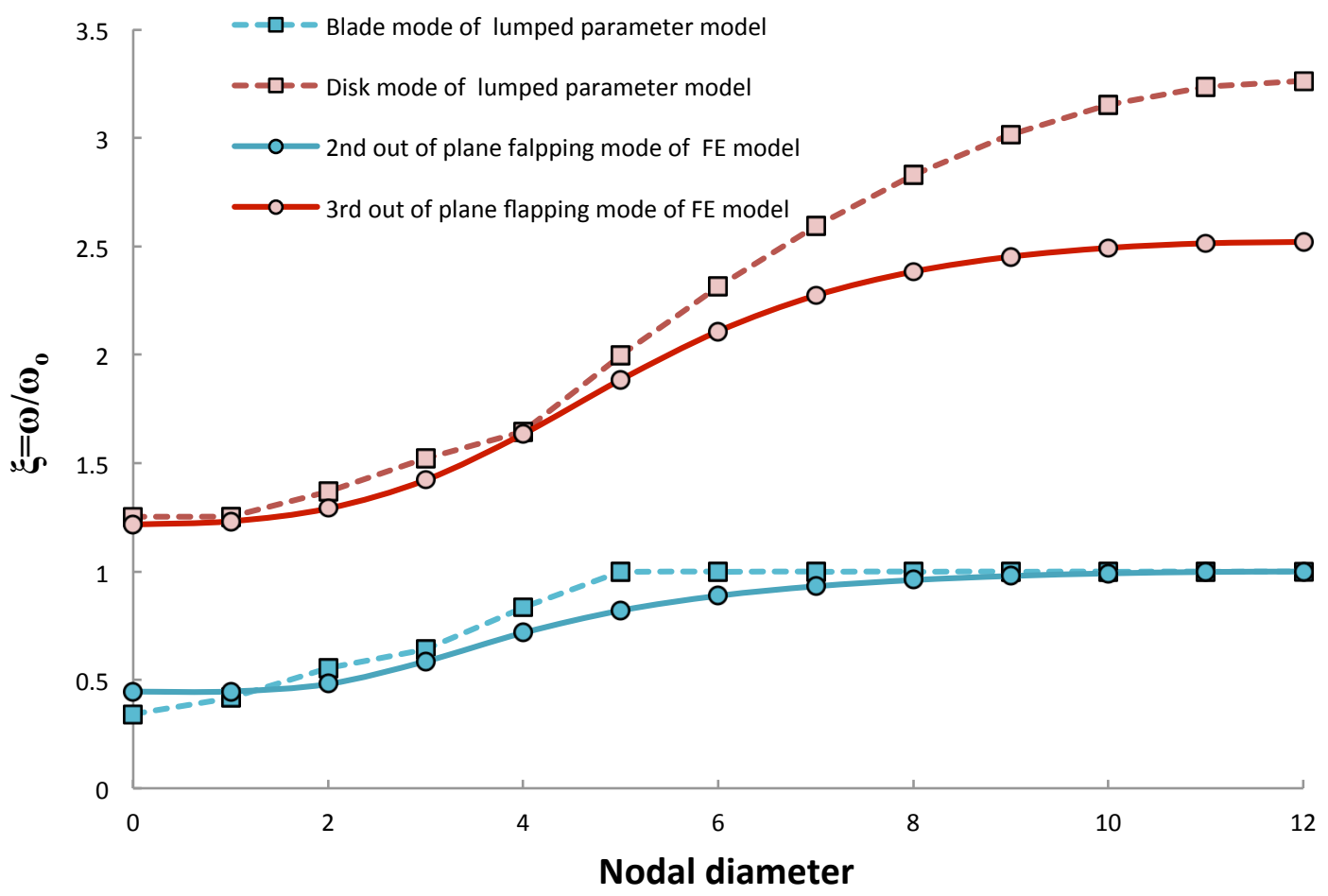

Figure 4 Comparisons of natural frequencies against nodal diameters between the lumped parameter and finite element models

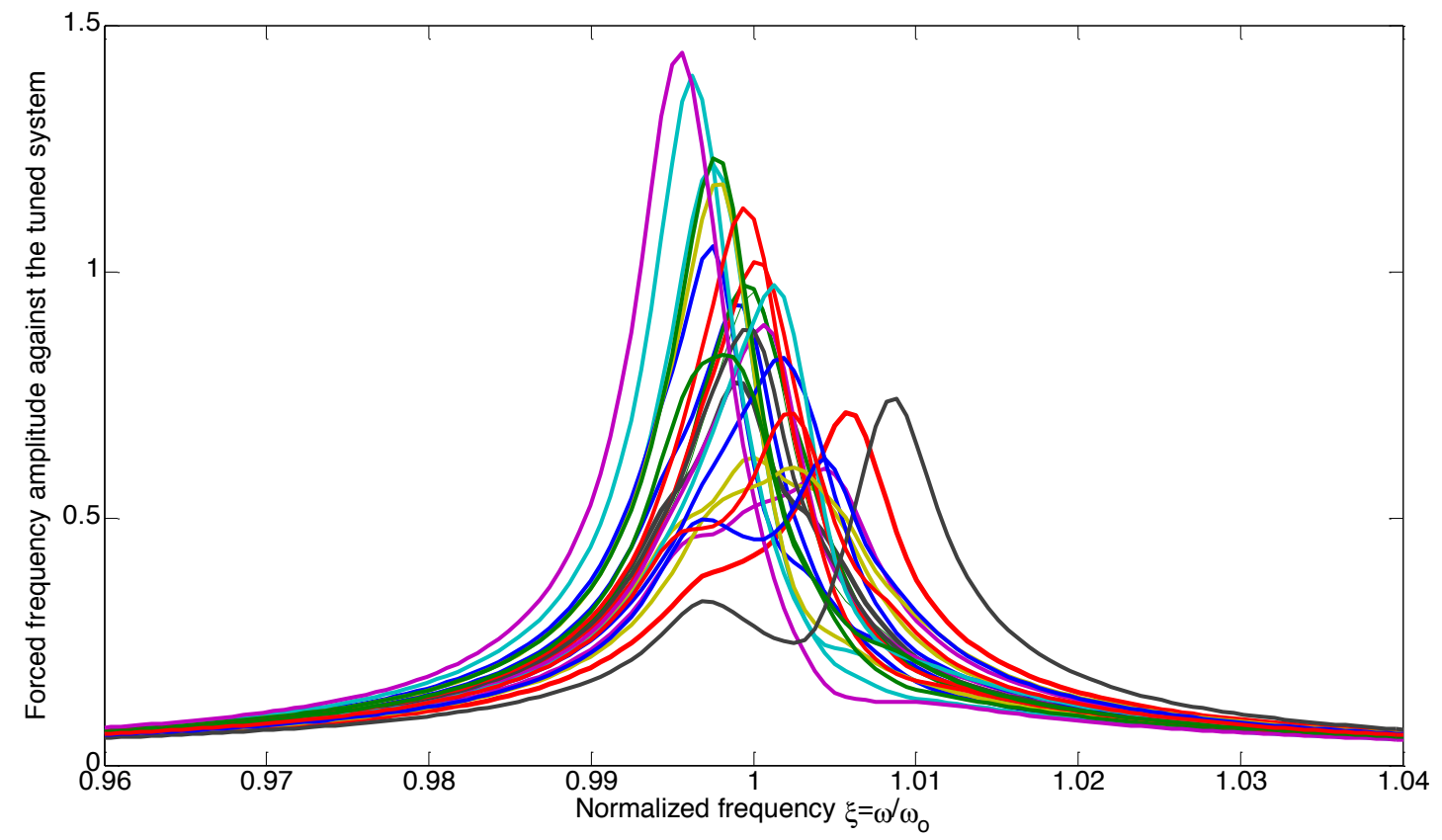


Figure 5 An example of blades frequency response from a mistuned bladed disc by the lumped parameter model
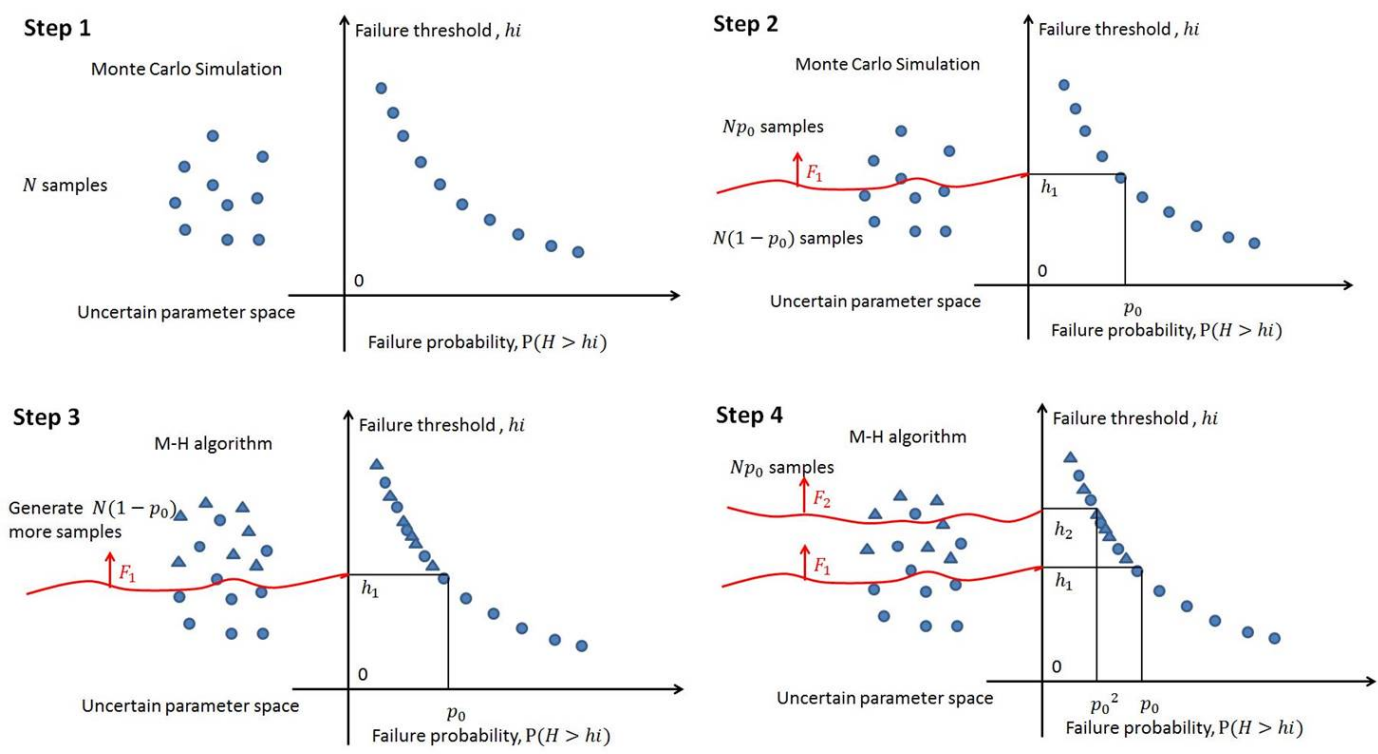

Figure 6 Illustration of the subset simulation method (SubSim) 


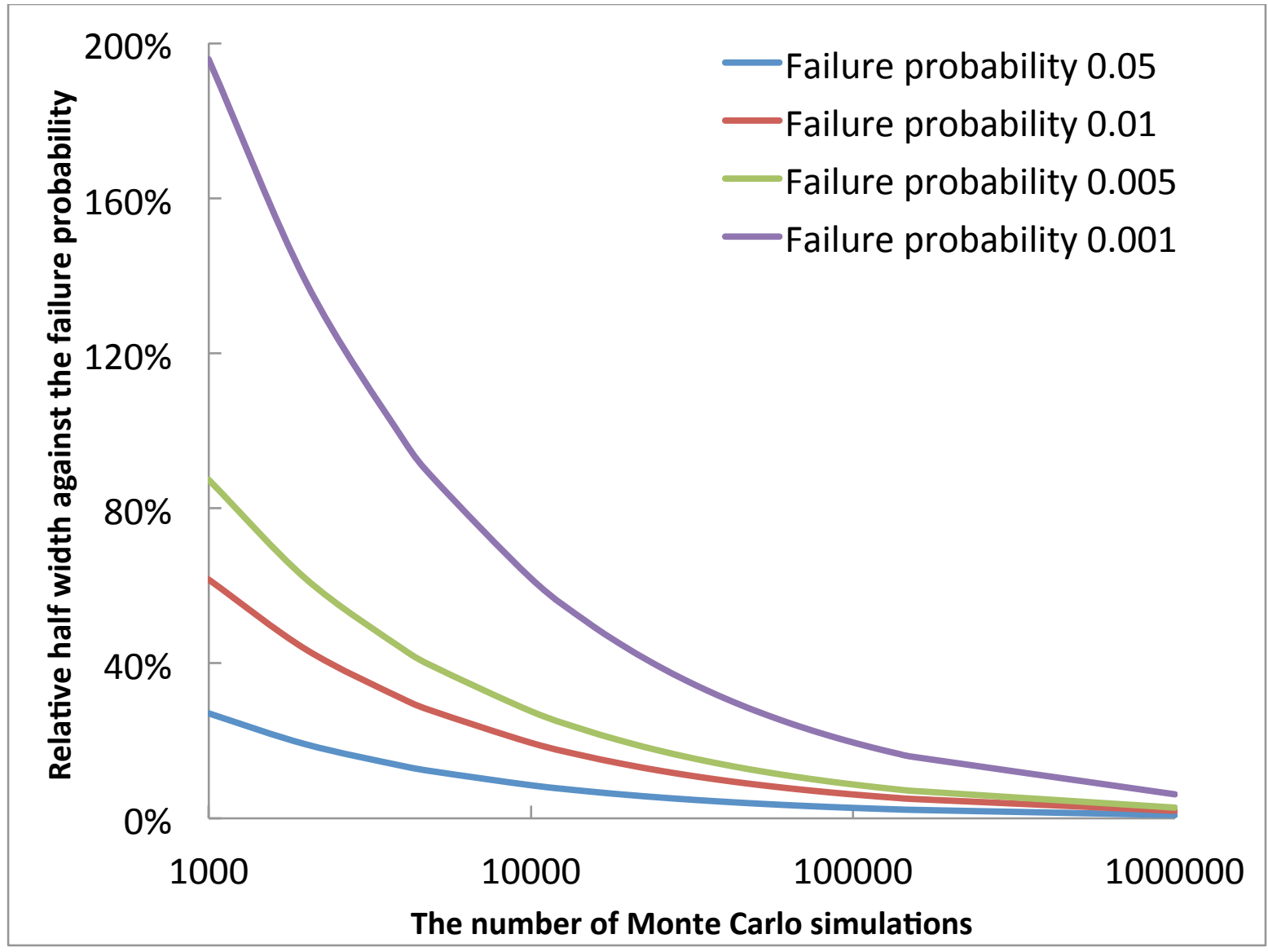

Figure 7 Variations of relative half width with sample size of MCS in different probability levels when $100(1-\alpha) \%=95 \%$

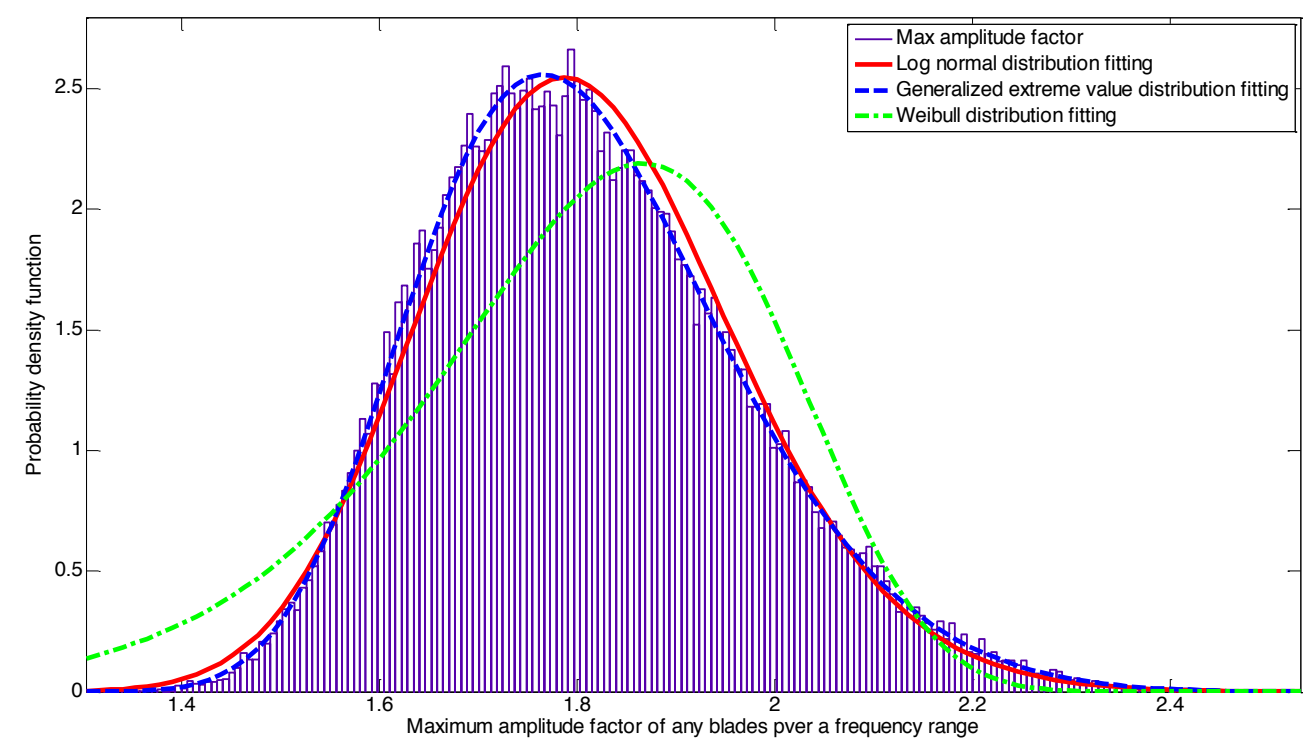

Figure 8 Probability density function of maximum amplitude factors 


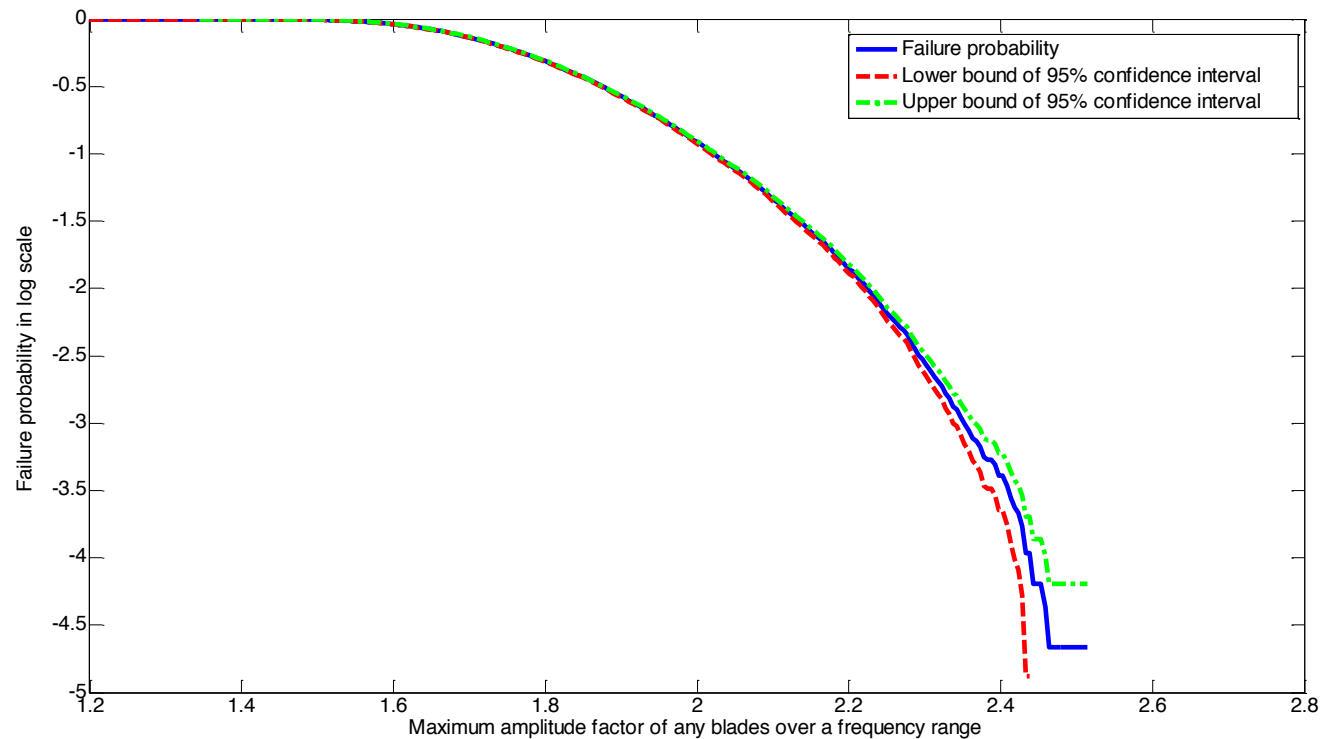

Figure 9 Failure probability distribution

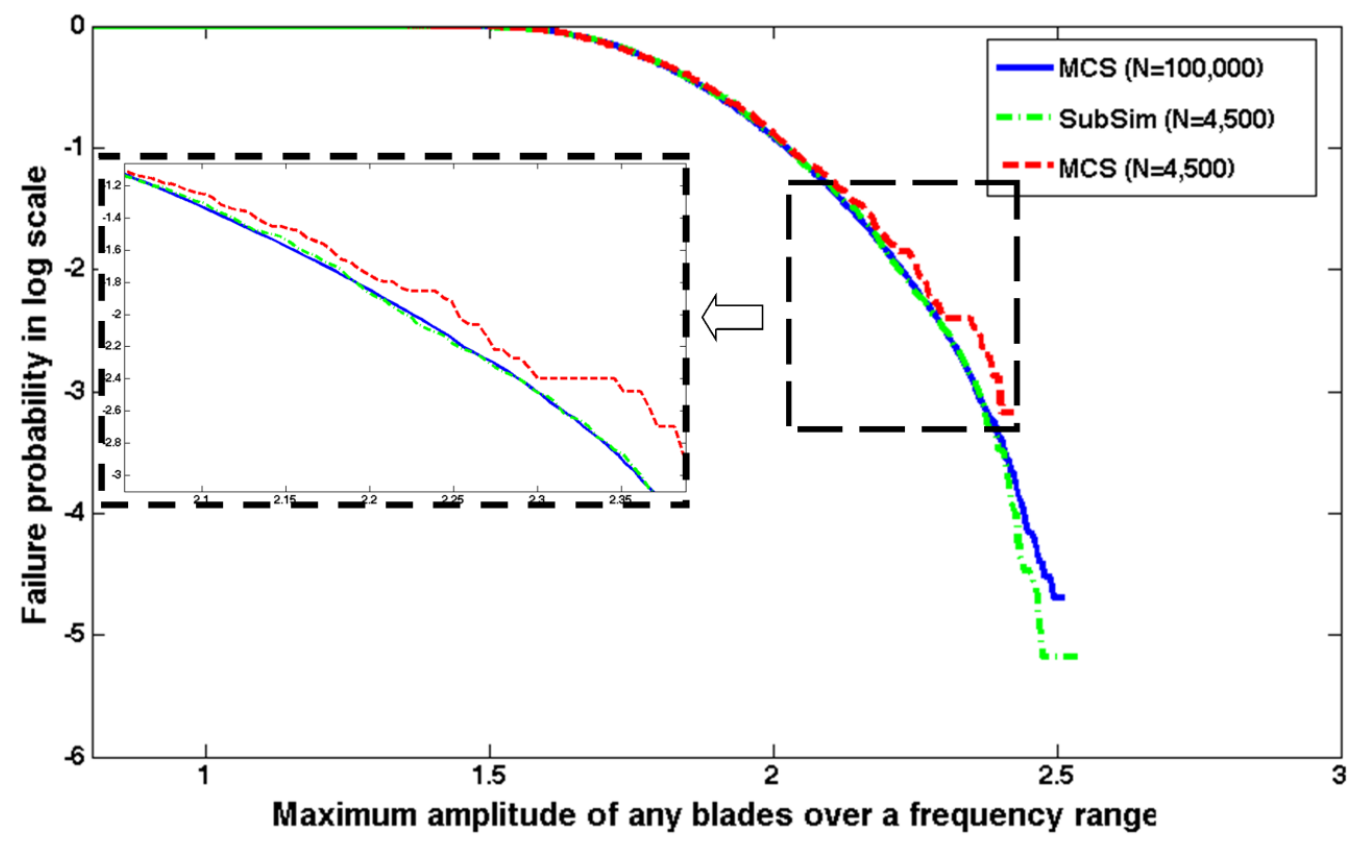

Figure 10 Failure probability distribution comparison between the SS and MCS 


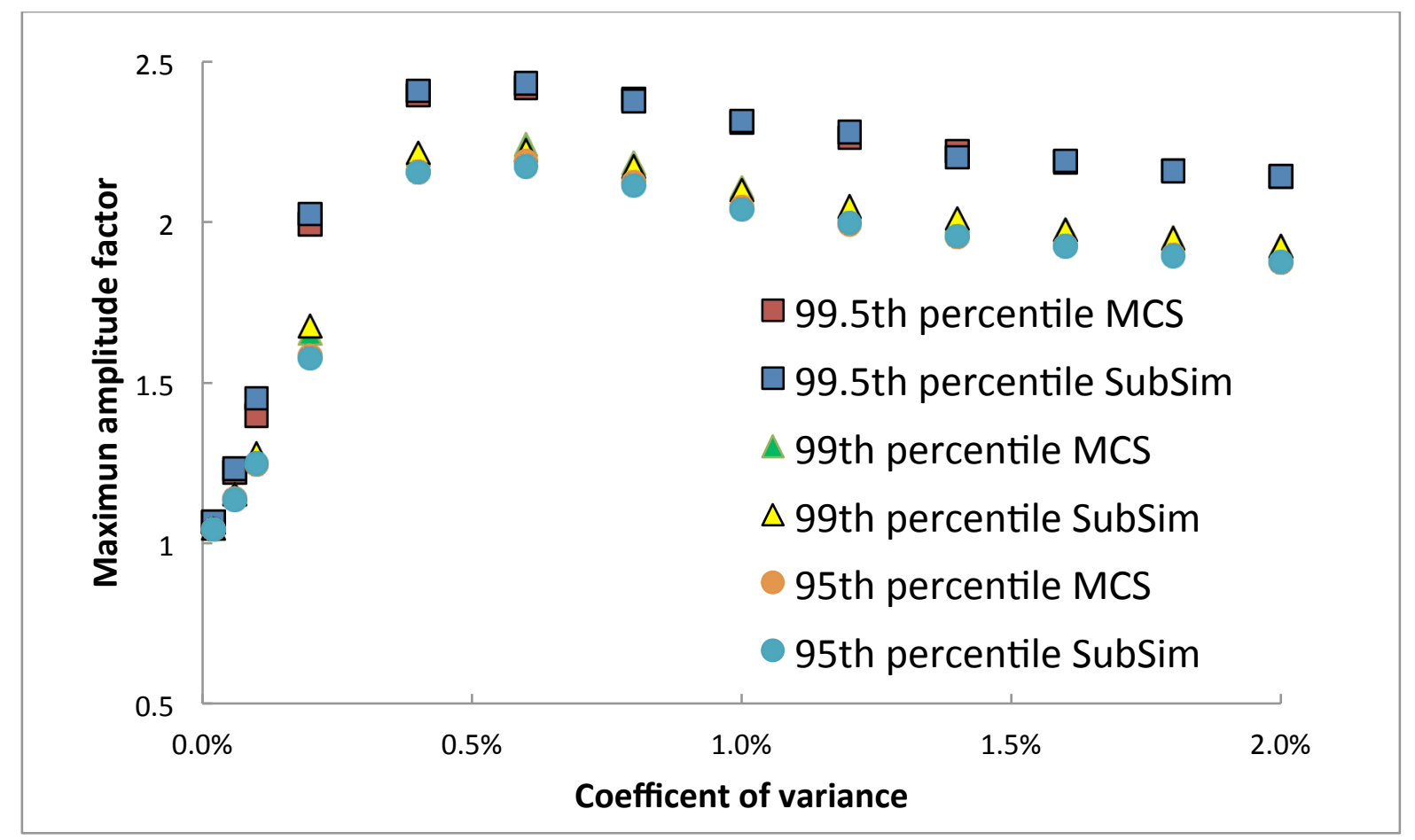

Figure 11 Maximum amplification factor for various coefficient of variance

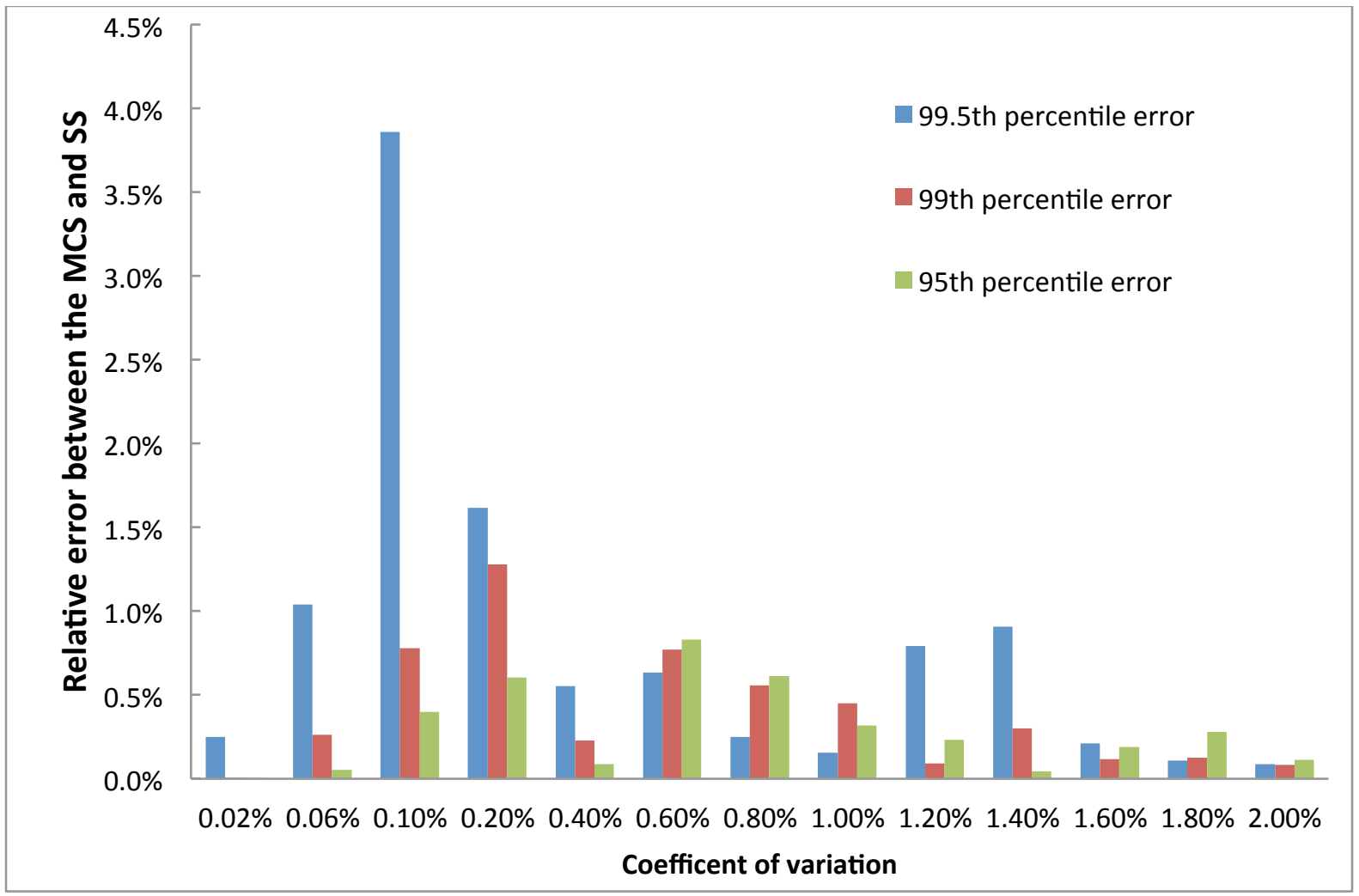

Figure 12 Relative error comparison for various coefficient of variance 


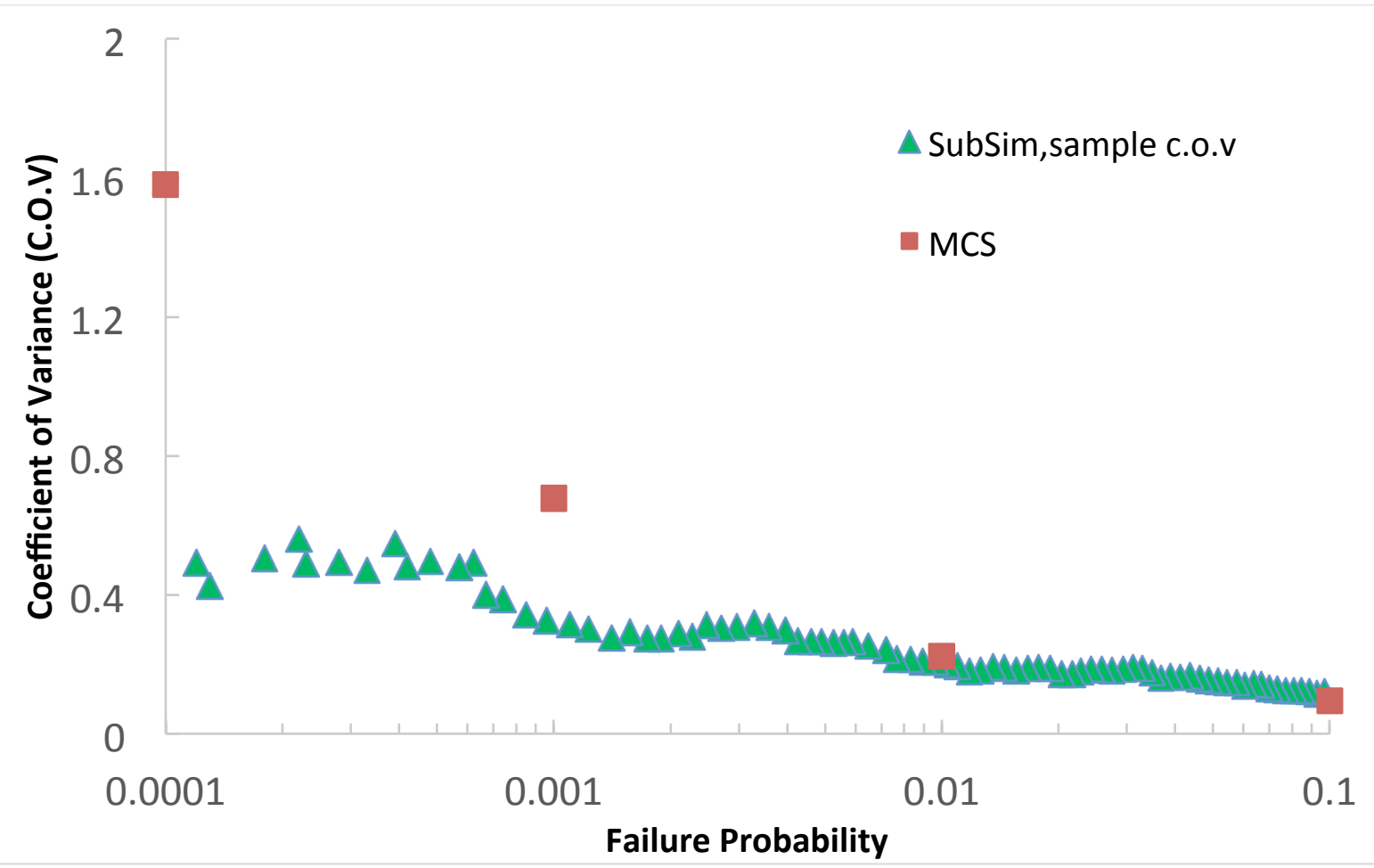

Figure 13 Coefficient of variation of failure probability

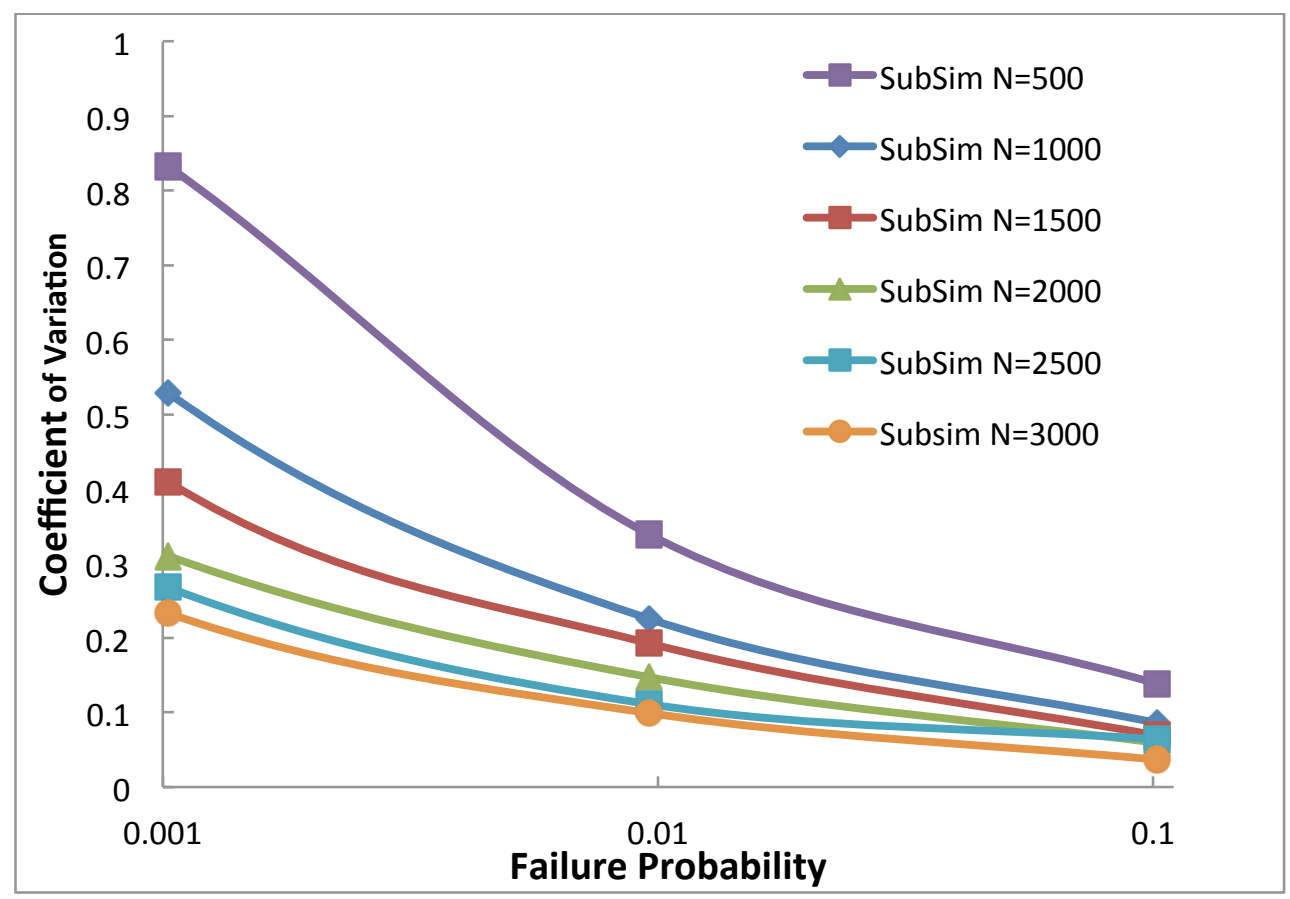

Figure 14 Coefficient of Variation comparisons with different conditional sample size 


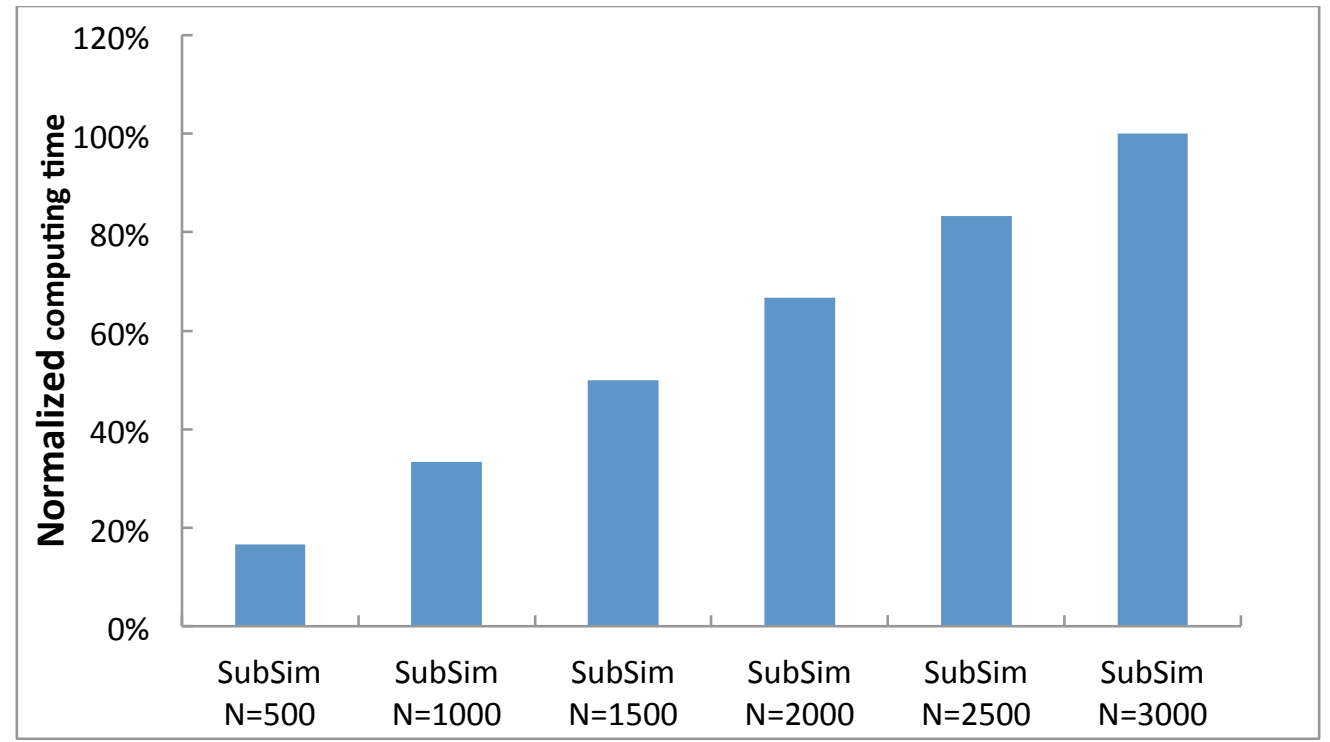

Figure 15 Normalized computing time comparisons in different conditional sample size 